\title{
NTRK gene amplification in patients with metastatic cancer
}

\author{
Su Jin Lee ${ }^{1 \star}$, Nayoung K. D. Kim ${ }^{2 \star}$, Se-Hoon Lee ${ }^{1 \star}$, Seung Tae Kim ${ }^{1}$, Se Hoon Park \\ Joon Oh Park ${ }^{1}$, Young Suk Park ${ }^{1}$, Ho Yeong Lim ${ }^{1}$, Won Ki Kang ${ }^{1}$, Woong Yang Park ${ }^{2}$, \\ Hee Jin Bang ${ }^{3}$, Kyoung-Mee Kim ${ }^{3}$, Keunchil Park' ${ }^{1}$, Jeeyun Lee ${ }^{1}$
}

${ }^{1}$ Division of Hematology-Oncology, Department of Medicine, Sungkyunkwan University School of Medicine, Seoul, Korea

${ }^{2}$ Samsung Genome Institute, Samsung Medical Center, Seoul, Korea

${ }^{3}$ Department of Pathology, Sungkyunkwan University School of Medicine, Seoul, Korea

Received: July 3, 2017

Revised: August 2, 2017

Accepted: August 3, 2017

\section{Corresponding author:}

Jeeyun Lee

Division of Hematology-

Oncology, Department of

Medicine, Samsung Medical

Center, Sungkyunkwan

University School of Medicine,

81 Irwon-ro, Gangnam-gu,

Seoul 06351, Korea

Tel: $+82-2-3410-1779$

E-mail: jyunlee@skku.edu

\begin{abstract}
Purpose: Neurotropic tropomyosin receptor kinase (NTRK) fusions have been identified in a variety of cancers, and tyrosine kinase inhibitors targeting the tropomyosin receptor kinase (TRK) receptor are currently in clinical trials. However, no reports are available on the effects of NTRK gene amplification.

Methods: Samples from patients enrolled in the sequencing program were analyzed using a next-generation sequencing (NGS) cancer panel. For cases in which NTRK amplification (defined as $\geq 4.0$ copies) was identified, panTRK immunohistochemical (IHC) staining of tissue microarrays was performed.

Results: A total of 1,250 tumor specimens collected between February 2014 and January 2016 were analyzed using the NGS cancer panel. NTRK amplification was detected in 28 cases of various types of cancer. Among 27 cases, only four were positive for panTRK IHC. These four cases were melanoma, sarcoma, lung cancer, and gastric cancer. We found that $2.2 \%$ of cancer patients showed NTRK amplification using NGS cancer panel and NTRK amplification resulted in protein overexpression in $14.8 \%$ of these patients.

Conclusion: Patients with NTRK amplification and increased TRK protein expression may be considered for inclusion in clinical trials for NTRK inhibitors.
\end{abstract}

Keywords: NGS cancer panel; NTRK gene amplification; TRK immunohistochemistry
This is an Open Access article distributed under the terms of the Creative Commons Attribution Non-Commercial License (http:// creativecommons.org/licenses/ by-nc/4.0/).

\section{INTRODUCTION}

Tropomyosin receptor kinase (TRK) is a receptor in the tyrosine kinase family that is activated by neurotrophins, a family of nerve growth factors [1-4]. Three members of the TRK family have been described: TRKA, TRKB, and TRKC, encoded by neurotropic tropomyosin receptor kinase 1 (NTRK1), NTRK2, and NTRK3, respectively. Trk family members play important roles in nervous system development through regulation of cell proliferation, differentiation, apoptosis, and sur- 
vival of neurons in both the central and peripheral nervous system. Trk receptors are expressed not only in the nervous system, but also in many other non-neuronal cell types and tissues, including monocytes, lung, bone, and pancreatic $\beta$ -cells $[5,6]$.

In 1982, the first NTRK1 gene fusion was identified in a colon cancer specimen; it contained sequence from tropomyosin 3 (TPM3; non-muscle tropomyosin) $[7,8]$. Subsequently, NTRK1 fusions have been detected at a frequency of $12 \%$ in papillary thyroid cancers, with TPM3-NTRK1 being the most common gene rearrangement [9-11]. In addition, TRKC and very recently TRKB have also been shown to form oncogenic chimeras in multiple tumor types [12,13]. Aside from gene fusions, only an in-frame deletion of NTRK1 in acute myeloid leukemia and a splice variant of NTRK1 in neuroblastoma have been functionally characterized as oncogenic to date [14-18]. However, no reports are available on the effects of NTRK gene amplification (defined as $\geq 4.0$ copies).

In the past several years, DNA sequencing technology has evolved dramatically. Next-generation DNA sequencing (NGS) has brought genome sequencing to clinical laboratories. The huge reduction in sequencing cost and the increase in sequencing efficiency, and the incomparable sequencing throughput, sensitivity, and accuracy all make NGS the most promising technology for cancer genomics and personalized cancer therapy. In this study, we investigated the prevalence of NTRK gene amplification using targeted sequencing and analyzed the association between gene amplification and TRK protein expression.

\section{METHODS}

\section{Patients}

This investigation was conducted in accordance with the ethical standards of the Declaration of Helsinki as well as national and international guidelines, and was approved by the Institutional Review Board at Samsung Medical Center, Seoul, Korea. Between October 2013 and January 2016, 1,250 patients with gastrointestinal cancer, lung cancer, and rare forms of cancer were prospectively enrolled in the NEXT-1, VIKTORY (NCT\#02299648), or LUNG PERSEQ (NCT\#02299622) trials at Samsung Medical Center that employed targeted sequencing (other cancer panels, such as lon Torrent [Thermo Fisher, Waltham, MA, USA], were excluded from this analysis). Patient inclusion criteria were as follows: age $\geq 18$ years, pathologically confirmed cancer, and availability of resection/biopsies of the primary or metastatic site and data on clinicopathologic characteristics.

\section{Targeted exome sequencing}

Genomic DNA was extracted, and a SureSelect customized kit (Agilent Technologies, Santa Clara, CA, USA) was used to capture 83 or 379 cancer-related genes, depending on the sequencing panel version. An Illumina HiSeq 2500 (Illumina, San Diego, CA, USA) was used for sequencing, and 100-bp paired-end reads were obtained. The sequencing reads were aligned to the human genome reference sequence (hg19) using BWA-mem (v0.7.5), SAMTOOLS (v0.1.18), Picard (v1.93), and GATK (v3.1.1) for sorting SAM/BAM files, duplicate marking, and local realignment, respectively. Local realignment and base recalibration were carried out using dbSNP137, Mills indels, HapMap, and Omni. Single nucleotide variants and indels were identified using Mutect (v1.1.4) and Pindel (v0.2.4), respectively. ANNOVAR was used to annotate the detected variants. Only variants with an allele frequency $>1 \%$ were included in the results. Copy number variation was calculated for the targeted sequencing regions by dividing read depth per exon by the normal reads per exon using an inhouse reference. Translocations in the target region were identified using an in-house algorithm (in preparation).

\section{PanTRK immunohistochemistry}

For tissue microarray construction, all H\&E stained slides were reviewed and the representative area was carefully selected and marked on all paraffin blocks. A 3-mm tissue core was taken from the representative region of each tumor specimen using Accumax (ISU Abxis, Seoul, Korea). Immunohistochemistry $(\mathrm{IHC})$ was performed under various conditions using five primary antibodies; panTRK (C17F1) from Cell Signaling (Danvers, MA, USA) was identified as a highly sensitive and specific primary antibody and was adopted for screening [19]. Mild cytoplasmic or membranous staining was considered to indicate a weakly positive result, and moderate to strong cytoplasmic staining was considered to indicate a positive result.

\section{Statistical analysis}

Student t-test was used to compare the means of continuous variables between the TRK-IHC-positive and -negative groups. $P$ values $<0.05$ were considered statistically significant. All statistical analyses were performed using SPSS software version 18.0 (SPSS Inc., Chicago, IL, USA). 


\section{RESULTS}

\section{Patients' characteristics}

Between October 2013 and January 2016, 1,250 patients with gastrointestinal cancer, lung cancer, or rare cancers were prospectively enrolled in the NEXT-1, VIKTORY (NCT\#02299648), or LUNG PERSEQ (NCT\#02299622) trials using targeted sequencing at Samsung Medical Center, Seoul, Korea. Among these 1,250 samples, we identified 28 cases with NTRK amplification (2.2\%), defined as $\geq 4.0$ copies (Fig. 1 ). Of 28 cases, almost all cases were NTRK1-amplified except for two cases of NTRK3 amplification and one case of NTRK2 amplification. The median age was 59 years (range, 23 to 74 years), and $60 \%$ of patients were male. These cases included lung cancer $(n=6)$, gastric cancer $(n=5)$, biliary tract cancer $(n=3)$, melanoma $(n=3)$, sarcoma $(n=3)$, pancreatic cancer $(n=2)$, hepatocellular carcinoma $(n=2)$, renal cell carcinoma $(n=2)$, bladder cancer $(n=1)$, and ovarian cancer $(n=1)$. The most common site of metastases was a non-regional/distant lymph node (LN; $n=14$ ) followed by lung, liver, peritoneal seeding, bone, pleura, pancreas, and brain. Baseline characteristics are listed in Table 1 and individual patients' information, with concomitant genetic aberrations, are also shown in Table 2 . The median NTRK copy number of all patients was 4.95 (range, 4.2 to 7.8 ).

\section{Identification of panTRK IHC-positive cases}

Tissue staining with TRK IHC was performed for 27 of the 28 cases (tissue was not available for the remaining case). Four of the 27 cases (14.8\%) were positive for TRK IHC (Fig. 2), and these four cases were all NTRK1-amplified. The first was a patient with acral melanoma, with an NTRK copy number of 6 and moderate to strong cytoplasmic TRK IHC staining. The second was a patient with sarcoma, with an NTRK copy number of 7.8 (the highest) and cytoplasmic TRK staining. The third was a patient with non-small cell lung cancer, with an

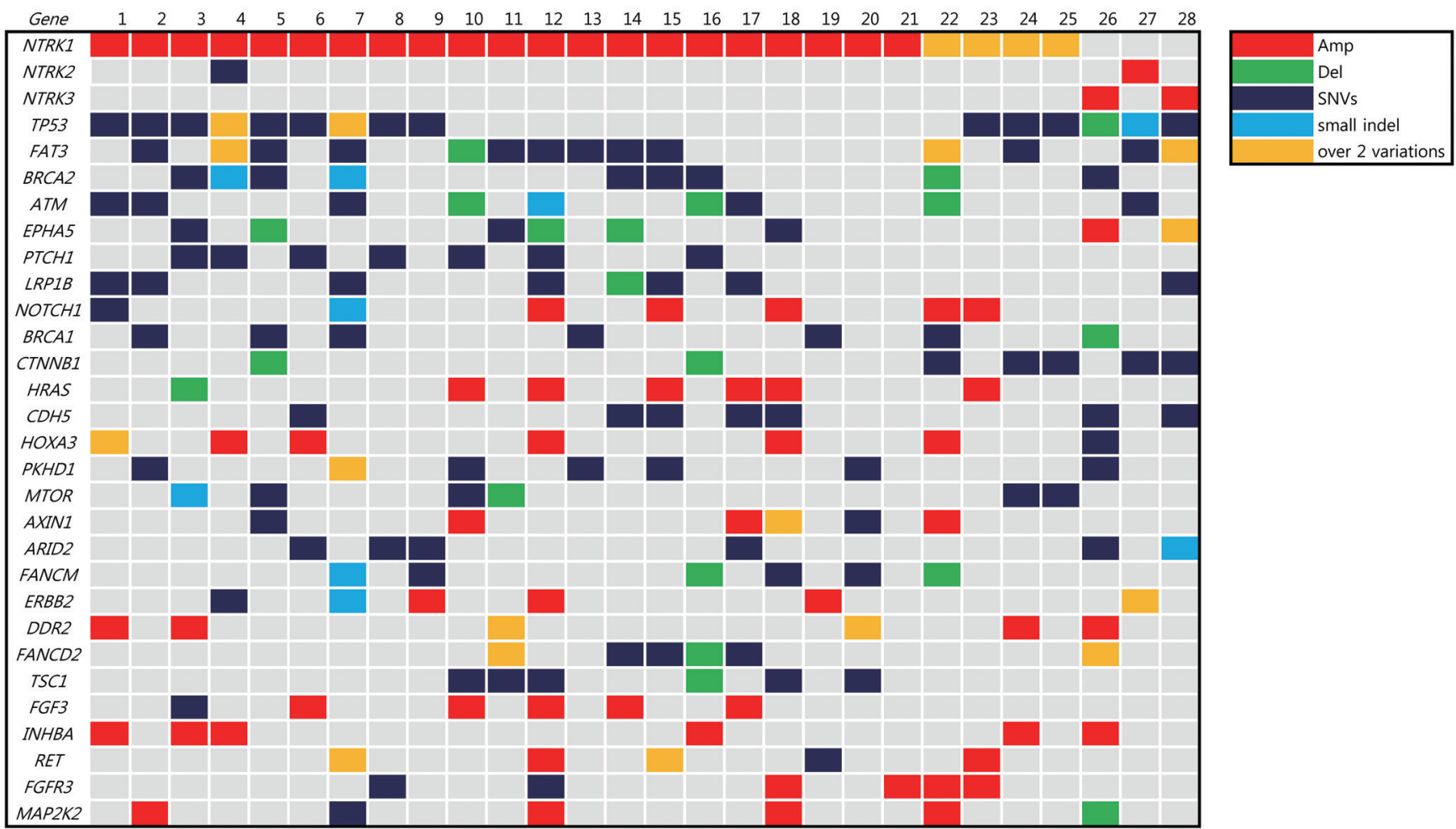

Fig. 1. Genomic landscape of cancer patients with neurotropic tropomyosin receptor kinase 1 (NTRK1) amplification. TP53, tumor protein p53; FAT3, FAT atypical cadherin 3; BRCA, breast cancer gene; EPHA5, EPH receptor A5; PTCH1, protein patched homolog 1; LRP1B, lowdensity lipoprotein receptor-related protein 1B; NOTCH, neurogenic locus notch homolog protein; CTNNB1, catenin beta-1; CDH5, cadherin 5; HOXA3, homeobox A3; PKHD1, polycystic kidney and hepatic disease 1; MTOR, mammalian target of rapamycin; AXIN1, axin 1; ARID2, ATrich interactive domain-containing protein 2; FANCM, Fanconi anemia, complementation group M; ERBB2, erb-b2 receptor tyrosine kinase 2; DDR2, discoidin domain receptor tyrosine kinase 2; FANCD2, Fanconi anemia complementation group D2; TSC1, tuberous sclerosis 1; FGF3, fibroblast growth factor 3; INHBA, inhibin, beta A; FGFR3, fibroblast growth factor receptor 3; MAP2K2, mitogen-activated protein kinase kinase 2; SNV, single nucleotide variant. 
Table 1. The characteristics of patients with NTRK amplification $(n=28)$

\begin{tabular}{|c|c|}
\hline Characteristic & Value \\
\hline Age (yr) & $59(2-74)$ \\
\hline \multicolumn{2}{|l|}{ Sex } \\
\hline Male & $17(60.7)$ \\
\hline Female & $11(39.3)$ \\
\hline \multicolumn{2}{|l|}{ Primary cancer site and histology } \\
\hline Lung cancer & $6(21.4)$ \\
\hline Adenocarcinoma & $3(10.7)$ \\
\hline Squamous cell carcinoma & $1(3.6)$ \\
\hline Large cell neuroendocrine carcinoma & $1(3.6)$ \\
\hline Small cell lung cancer & $1(3.6)$ \\
\hline Gastric cancer & $5(17.9)$ \\
\hline Adenocarcinoma & $2(7.1)$ \\
\hline Signet ring cell carcinoma & $2(7.1)$ \\
\hline Poorly differentiated neuroendocrine carcinoma & $1(3.6)$ \\
\hline Biliary tract cancer (all adenocarcinoma) & $3(10.7)$ \\
\hline Hilar cholangiocarcinoma & $1(3.6)$ \\
\hline Intrahepatic cholangiocarcinoma & $1(3.6)$ \\
\hline Ampulla of Vater cancer & $1(3.6)$ \\
\hline Melanoma & $3(10.7)$ \\
\hline Mucosal & $2(7.1)$ \\
\hline Acral & $1(3.6)$ \\
\hline Sarcoma & $3(10.7)$ \\
\hline Uterine leiomyosarcoma & $1(3.6)$ \\
\hline PEComa & $1(3.6)$ \\
\hline Unspecified & $1(3.6)$ \\
\hline Pancreatic cancer & $2(7.1)$ \\
\hline Acinar cell carcinoma & $2(7.1)$ \\
\hline Adenocarcinoma & $2(7.1)$ \\
\hline Hepatocellular carcinoma & $2(7.1)$ \\
\hline Renal cell carcinoma (all clear cell) & $2(7.1)$ \\
\hline Bladder cancer, TCC & $1(3.6)$ \\
\hline Ovarian cancer & $1(3.6)$ \\
\hline \multicolumn{2}{|l|}{ Initial stage } \\
\hline Loco-regional disease & $15(5.6)$ \\
\hline Metastatic disease & $1(46.4)$ \\
\hline \multicolumn{2}{|l|}{ No. of prior systemic treatment regimen } \\
\hline 1 & $9(2.1)$ \\
\hline 2 & $10(5.7)$ \\
\hline 3 & $4(14.3)$ \\
\hline$\geq 4$ & $5(17.9)$ \\
\hline \multicolumn{2}{|l|}{ Site of distant metastasis } \\
\hline Lymph node & $14(50.0)$ \\
\hline Lung & $12(42.9)$ \\
\hline Liver & $8(28.6)$ \\
\hline Peritoneal seeding & $7(25.0)$ \\
\hline Bone & $4(14.3)$ \\
\hline Pleura & $3(10.7)$ \\
\hline Pancreas & $2(7.1)$ \\
\hline Brain & $2(7.1)$ \\
\hline
\end{tabular}

Values are presented as median (range) or number (\%). NTRK, neurotropic tropomyosin receptor kinase; PEComa, perivascular epithelioid cell tumor; TCC, transitional cell carcinoma.

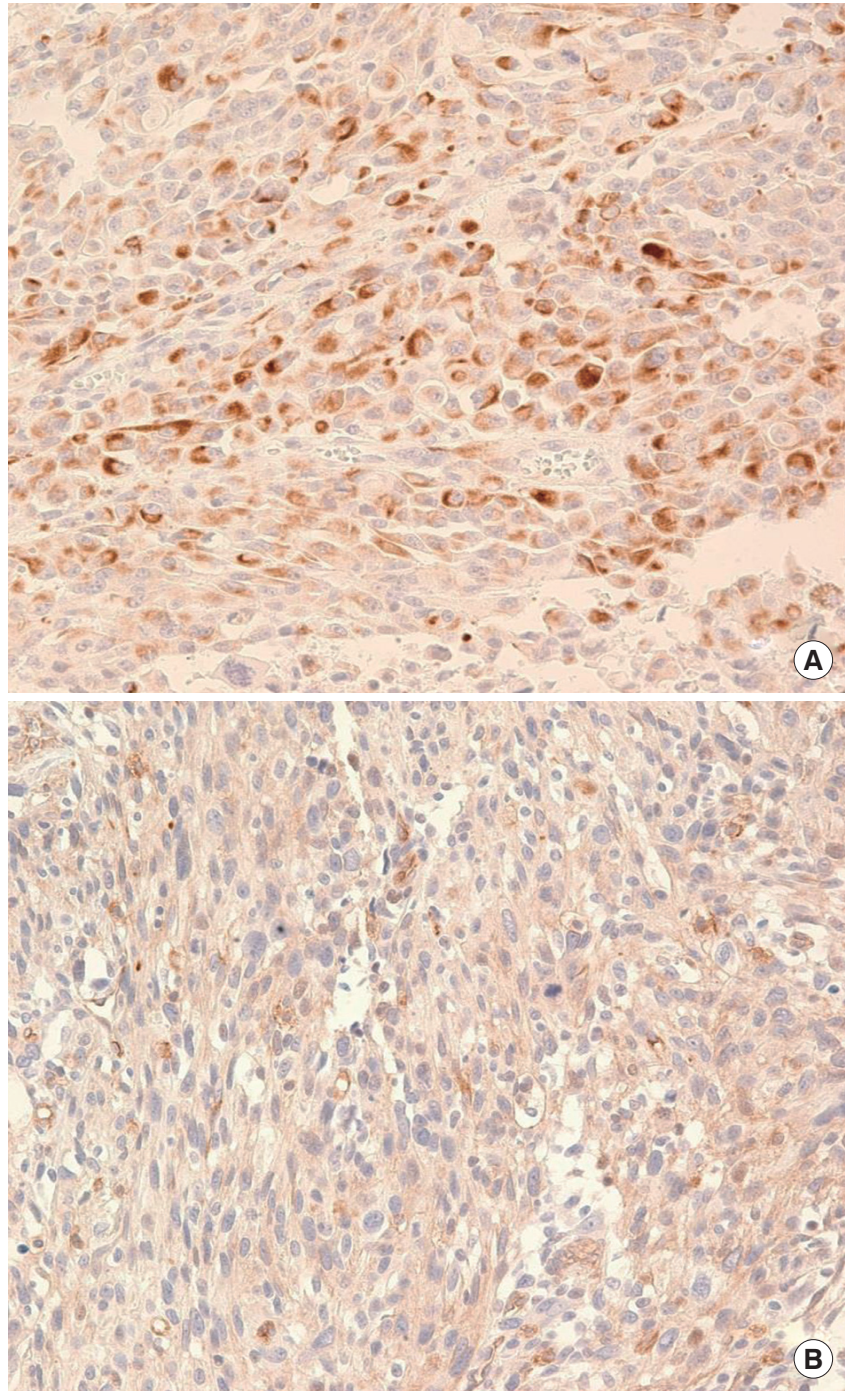

Fig. 2. Representative tropomyosin receptor kinase immunohistochemical staining $(\times 400)$. (A) Acral melanoma with moderate to strong cytoplasmic immunohistochemical staining. (B) Sarcoma with mild cytoplasmic staining.

NTRK copy number of 4.6 and mild cytoplasmic staining. The last case was a patient with gastric cancer, with an NTRK copy number of 4.6. The median NTRK copy number in TRK IHCnegative versus positive cases was 4.95 vs. 5.3 , but the difference was not significant $(P=0.509)$

\section{Characteristics of patients showing NTRK amplifica- tion and overexpression}

The first case that showed NTRK amplification is a 60-yearold male patient who initially presented with stage II acral melanoma of the big toe and underwent a curative resection in 2012. He recurred with inguinal LN metastasis and underwent an inguinal LN dissection in 2014. One year later, new inguinal, external iliac, and obturator LN metastases devel- 


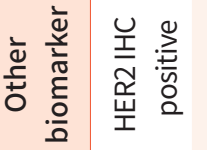

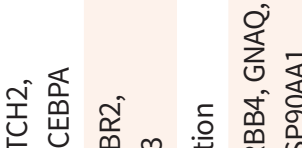

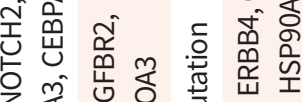

б z z⿺⿻一⿰冫⿰亅⿱丿丶丶⿱⿰㇒一乂

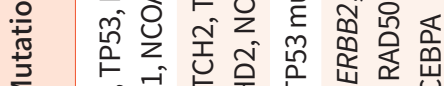

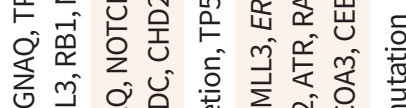

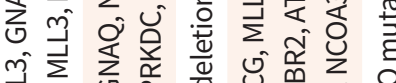

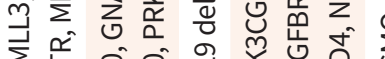

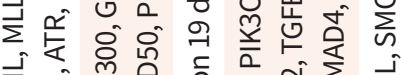

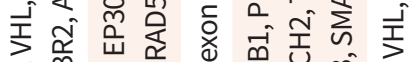

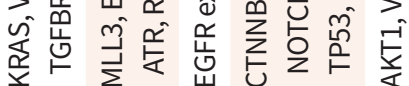

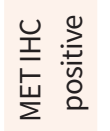

กิบ

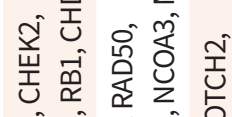

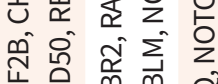

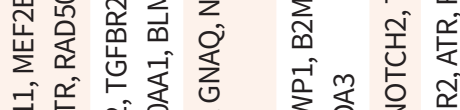

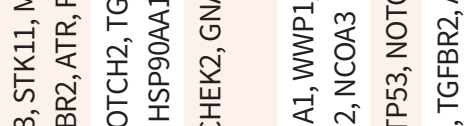

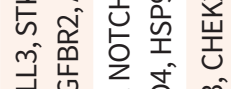

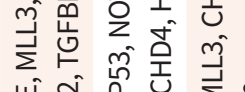

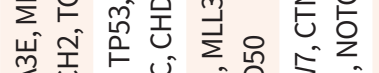

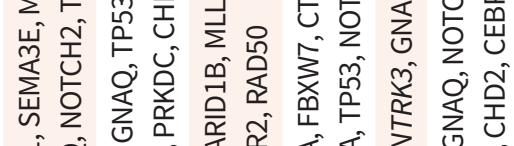

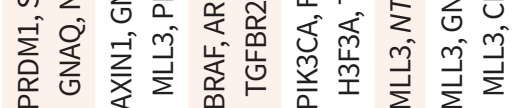

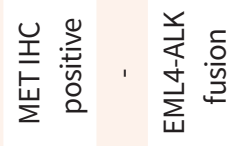

ֻٕ

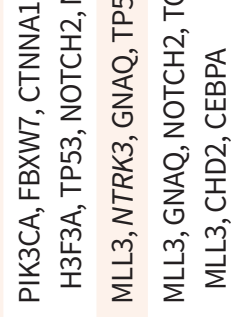

呈悹

高 产

m

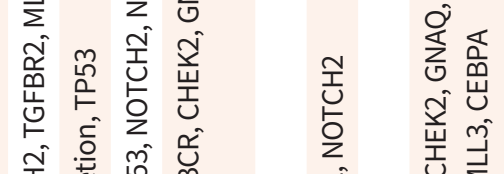

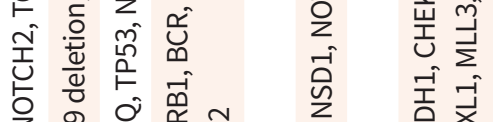

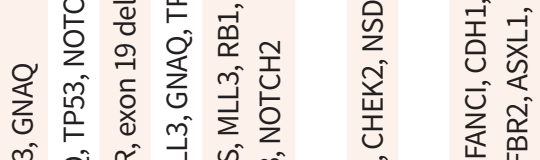

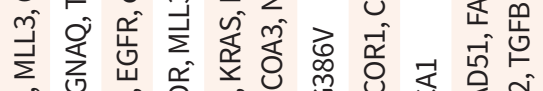

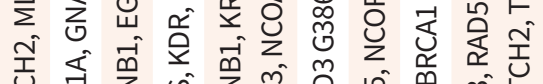

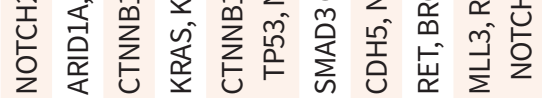

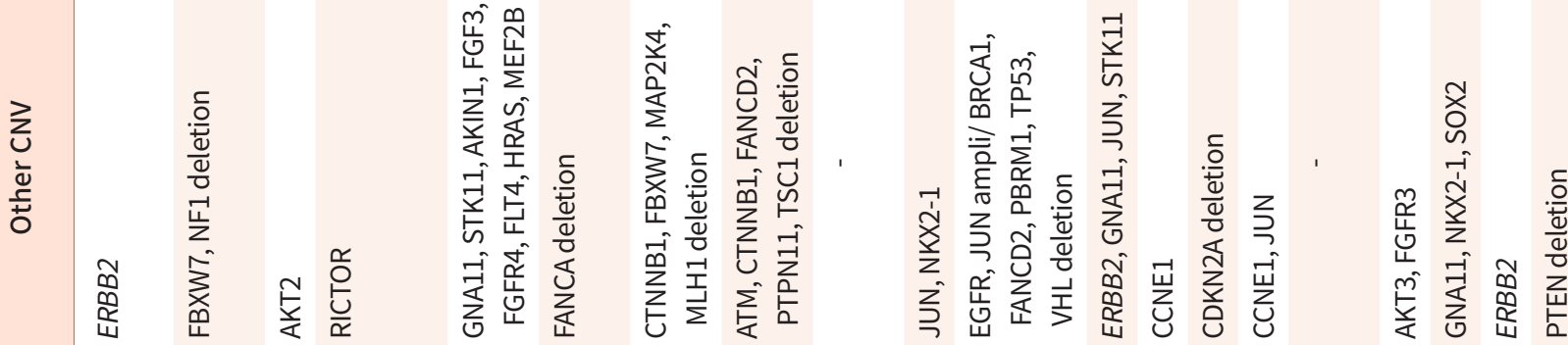

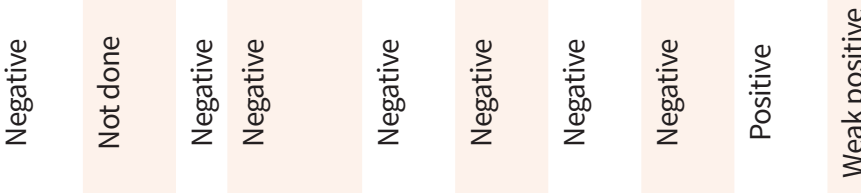

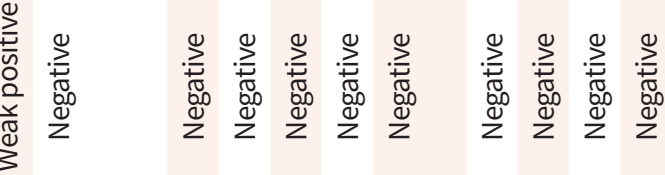

蛋

항

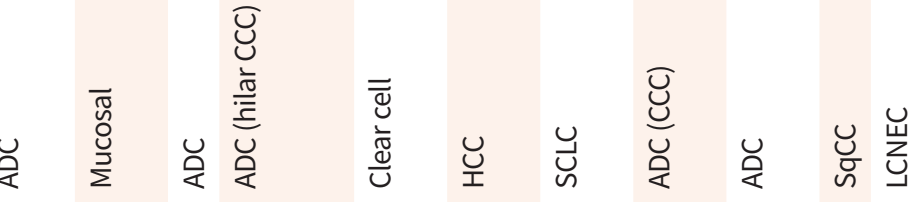

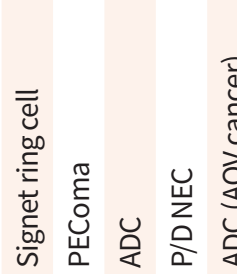

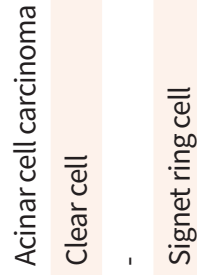

离

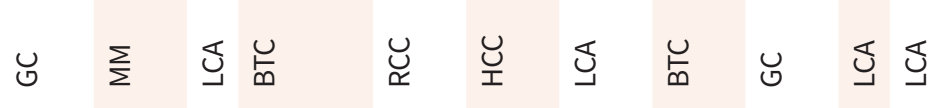

ن ৩

萿 8 ก ก

$\frac{\frac{0}{0}}{\frac{0}{\sigma}}$

๖

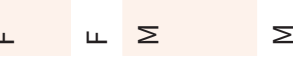

$\leftarrow \quad \Sigma$

$\Sigma \Sigma \Sigma \Sigma$

$\Sigma \Sigma L \Sigma \Sigma \Sigma L \sqcup \Sigma$ 


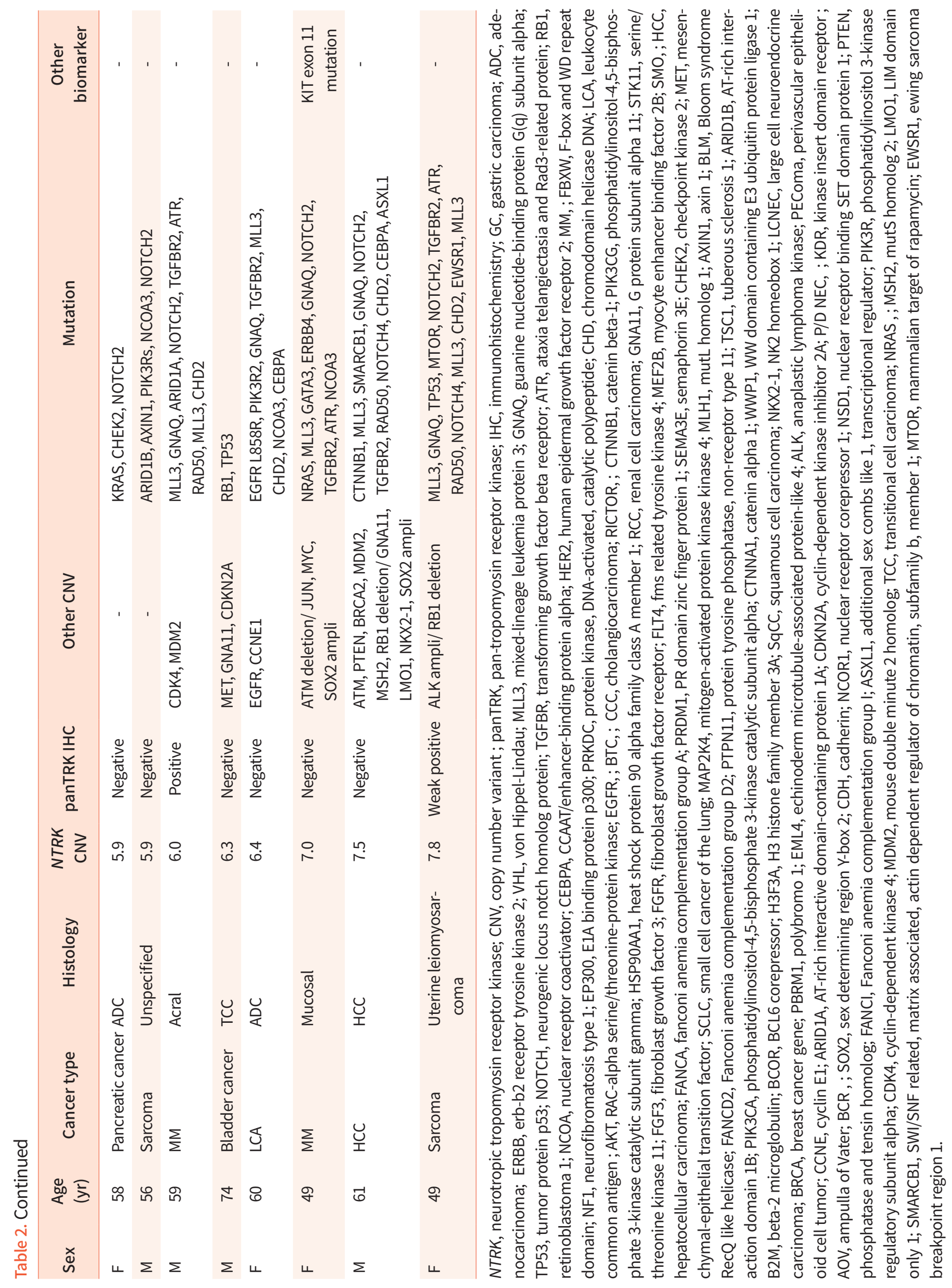


oped and he was referred to our center. After confirmation that the patient possessed wild-type BRAF and KIT, he received nine cycles of palliative dacarbazine/cisplatin/tamoxifen chemotherapy with partial response, but the disease continued to progress. We plan to treat him with second-line chemotherapy.

The second NTRK-amplified patient is a 50-year-old female who underwent a total hysterectomy for uterine leiomyosarcoma in 2001. The tumor recurred in 2013 and she underwent radiofrequency ablation for muscle metastasis and a metastasectomy for lung metastasis. But 5 months later, multiple bone, pancreas, and lung metastases developed and she was treated with several lines of chemotherapy as well as palliative radiotherapy for bone metastasis. She has now joined a clinical trial for a new tyrosine kinase inhibitor (TKI) that targets TRK.

The third patient was a 49-year-old male with squamous cell lung cancer that was resected in 2012 after neoadjuvant chemoradiation for stage IIIA. Nine months later, supraclavicular LN metastasis developed and he underwent salvage chemoradiation followed by palliative chemotherapy and radiotherapy for progression. In 2015, he underwent a craniotomy and tumor removal for cerebellar metastasis, but died due to disease progression.

The fourth patient is a 65-year-old male with gastric adenocarcinoma who was initially diagnosed with stage IV cancer and who has been treated with third-line chemotherapy.

\section{DISCUSSION}

Gene amplification is defined as an increase in the copy number of a restricted region of a chromosome arm [20,21]. Gene amplification is an influential factor in the expression of both protein-coding and non-coding genes, affecting the activity of various signaling pathways in cancer. Gene amplification, similar to gene mutation, plays a significant role in tumorigenesis in many types of cancer, such as gastric cancer, ovarian cancer, hepatocellular carcinoma, colon cancer, and others [22,23]. Thus, targeting the "driver genes" that are amplified may provide novel opportunities for precision medicine [20].

One of the most studied gene amplifications is erb-b2 receptor tyrosine kinase 2 (ERBB2), an important driver oncogene for breast cancer [24]. In breast cancer, gene amplification of $E R B B 2$ is strongly correlated with its protein expression. Moreover, ERBB2 amplification is also observed in gastric cancer $[25,26]$. Mesenchymal-epithelial transition factor
(MET) is a proto-oncogene that encodes a receptor tyrosine kinase, and aberrant activation of MET signaling occurs in a subset of advanced cancers as a result of various genetic alterations, including gene amplification [27]. Recently, MET amplification was identified as a potential oncogenic driver for several cancers, and therapy with TKIs that target MET shows promise as an effective treatment, based on preclinical and clinical data [28].

Using gene panel analysis, we showed that $2.2 \%$ of cancer patients had NTRK amplification, and that NTRK amplification resulted in protein overexpression in $14.8 \%$ of these patients. The results of gene amplification detected frequently conflict with the results of the corresponding protein overexpression. For example in TOGA trial [29], 131 patients (22.4\%) were ERBB2 IHC 0 or 1 with gene amplification by fluorescent in situ hybridization. This discrepancy can be explained by decreased internalization or turnover of the HER2 (human epidermal growth factor receptor 2) protein [30] and bystander effect of ERBB2 gene amplification [31]. Ma et al. [32] recently suggested that interactions with peripheral blood mononuclear cells in the tumor microenvironment might increase ERBB2 and MMP9 (matrix metallopeptidase 9) mRNA and it might be involved in the mechanism responsible for this discrepancy. In comparison with ERBB2 amplification, protein overexpression was much lower in NTRK-amplified cancers. To determine whether or not NTRK amplification is an oncogenic driver, further preclinical study using TRK TKIs or RNA interference targeted to NTRK mRNA is needed. Even though we cannot conclude that NTRK amplification is an oncogenic driver, our study suggests that NTRK TKIs show promise to provide an effective treatment for cancers involving NTRK amplification.

Currently, several TKIs with activity against the TRK family, such as entrectinib (NCT\#02097810) or LOXO-101 (NCT\#0257 6431), are being investigated in clinical trials. Clinical efficacy has been reported in patients with well-known NTRK fusions, such as TPM3-NTRK1, LMNA-NTRK1, or ETV6-NTRK3 fusions [33-37]. Although the clinical implications of NTRK amplification, in terms of responsiveness to TRK inhibitors, has yet to be demonstrated, our data indicate that patients with NTRK amplification that show TRK protein expression may be considered for inclusion in clinical trials for TRK inhibitors.

\section{CONFLICTS OF INTEREST}

No potential conflict of interest relevant to this article was reported. 


\section{ACKNOWLEDGMENTS}

This work was supported by a grant from the Korean Health Technology R\&D Project, Ministry of Health and Welfare, Republic of Korea (HI14C2188, HI14C3418). Support was also provided by a grant from the 20 by 20 project of Samsung Medical Center (GF01140111). The funders had no role in the design and conduct of the study.

\section{REFERENCES}

1. Ardini E, Bosotti R, Borgia AL, De Ponti C, Somaschini A, Cammarota $\mathrm{R}$, et al. The TPM3-NTRK1 rearrangement is a recurring event in colorectal carcinoma and is associated with tumor sensitivity to TRKA kinase inhibition. Mol Oncol 2014;8:1495-507.

2. Barbacid M. Neurotrophic factors and their receptors. Curr Opin Cell Biol 1995;7:148-55.

3. Kaplan DR, Martin-Zanca D, Parada LF. Tyrosine phosphorylation and tyrosine kinase activity of the trk proto-oncogene product induced by NGF. Nature 1991;350: 158-60.

4. Levi-Montalcini R. The nerve growth factor 35 years later. Science 1987;237:1154-62.

5. Vaishnavi A, Le AT, Doebele RC. TRKing down an old oncogene in a new era of targeted therapy. Cancer Discov 2015;5:25-34.

6. Coppola V, Barrick CA, Southon EA, Celeste A, Wang K, Chen $B$, et al. Ablation of TrkA function in the immune system causes B cell abnormalities. Development 2004; 131:5185-95.

7. Pulciani S, Santos E, Lauver AV, Long LK, Aaronson SA, Barbacid M. Oncogenes in solid human tumours. Nature 1982;300:539-42.

8. Martin-Zanca D, Hughes SH, Barbacid M. A human oncogene formed by the fusion of truncated tropomyosin and protein tyrosine kinase sequences. Nature 1986;319:7438.

9. Greco A, Miranda C, Pagliardini S, Fusetti L, Bongarzone I, Pierotti MA. Chromosome 1 rearrangements involving the genes TPR and NTRK1 produce structurally different thyroid-specific TRK oncogenes. Genes Chromosomes Cancer 1997;19:112-23.

10. Alberti L, Carniti C, Miranda C, Roccato E, Pierotti MA. RET and NTRK1 proto-oncogenes in human diseases. J Cell Physiol 2003;195:168-86.

11. Greco A, Miranda C, Pierotti MA. Rearrangements of
NTRK1 gene in papillary thyroid carcinoma. Mol Cell Endocrinol 2010;321:44-9.

12. Jones DT, Hutter B, Jager N, Korshunov A, Kool M, Warnatz $\mathrm{HJ}$, et al. Recurrent somatic alterations of FGFR1 and NTRK2 in pilocytic astrocytoma. Nat Genet 2013;45: 927-32.

13. Rubin BP, Chen CJ, Morgan TW, Xiao S, Grier HE, Kozakewich HP, et al. Congenital mesoblastic nephroma t(12;15) is associated with ETV6-NTRK3 gene fusion: cytogenetic and molecular relationship to congenital (infantile) fibrosarcoma. Am J Pathol 1998;153:1451-8.

14. Tacconelli A, Farina AR, Cappabianca L, Desantis G, Tessitore A, Vetuschi A, et al. TrkA alternative splicing: a regulated tumor-promoting switch in human neuroblastoma. Cancer Cell 2004;6:347-60.

15. Reuther GW, Lambert QT, Caligiuri MA, Der CJ. Identification and characterization of an activating TrkA deletion mutation in acute myeloid leukemia. Mol Cell Biol 2000; 20:8655-66.

16. Light JE, Koyama H, Minturn JE, Ho R, Simpson AM, lyer R, et al. Clinical significance of NTRK family gene expression in neuroblastomas. Pediatr Blood Cancer 2012;59:22632.

17. Bapat AA, Hostetter G, Von Hoff DD, Han H. Perineural invasion and associated pain in pancreatic cancer. Nat Rev Cancer 2011;11:695-707.

18. Thiele CJ, Li Z, McKee AE. On Trk: the TrkB signal transduction pathway is an increasingly important target in cancer biology. Clin Cancer Res 2009;15:5962-7.

19. Lee SJ, Li GG, Kim ST, Hong ME, Jang J, Yoon N, et al. NTRK1 rearrangement in colorectal cancer patients: evidence for actionable target using patient-derived tumor cell line. Oncotarget 2015;6:39028-35.

20. Matsui A, Ihara T, Suda H, Mikami H, Semba K. Gene amplification: mechanisms and involvement in cancer. Biomol Concepts 2013;4:567-82.

21. Albertson DG. Gene amplification in cancer. Trends Genet 2006;22:447-55.

22. Leary RJ, Lin JC, Cummins J, Boca S, Wood LD, Parsons DW, et al. Integrated analysis of homozygous deletions, focal amplifications, and sequence alterations in breast and colorectal cancers. Proc Natl Acad Sci U S A 2008;105: 16224-9.

23. Xu H, Zhu X, Xu Z, Hu Y, Bo S, Xing T, et al. Non-invasive analysis of genomic copy number variation in patients with hepatocellular carcinoma by next generation DNA sequencing. J Cancer 2015;6:247-53. 
24. Slamon DJ, Clark GM, Wong SG, Levin WJ, Ullrich A, McGuire WL. Human breast cancer: correlation of relapse and survival with amplification of the HER-2/neu oncogene. Science 1987;235:177-82.

25. Kauraniemi P, Kallioniemi A. Activation of multiple cancer-associated genes at the ERBB2 amplicon in breast cancer. Endocr Relat Cancer 2006;13:39-49.

26. Yano T, Doi T, Ohtsu A, Boku N, Hashizume K, Nakanishi M, et al. Comparison of HER2 gene amplification assessed by fluorescence in situ hybridization and HER2 protein expression assessed by immunohistochemistry in gastric cancer. Oncol Rep 2006;15:65-71.

27. Liu X, Newton RC, Scherle PA. Developing c-MET pathway inhibitors for cancer therapy: progress and challenges. Trends Mol Med 2010;16:37-45.

28. Kawakami H, Okamoto I, Okamoto W, Tanizaki J, Nakagawa K, Nishio K. Targeting MET amplification as a new oncogenic driver. Cancers (Basel) 2014;6:1540-52.

29. Bang YJ, Van Cutsem E, Feyereislova A, Chung HC, Shen L, Sawaki A, et al. Trastuzumab in combination with chemotherapy versus chemotherapy alone for treatment of HER2-positive advanced gastric or gastro-oesophageal junction cancer (ToGA): a phase 3, open-label, randomized controlled trial. Lancet 2010;376:687-97.

30. Kuesters S, Maurer M, Burger AM, Metz T, Fiebig HH. Correlation of ErbB2 gene status, mRNA and protein expression in a panel of $>100$ human tumor xenografts of different origin. Onkologie 2006;29:249-56.

31. Moelans CB, van Diest PJ, Milne AN, Offerhaus GJ. Her-2/ neu testing and therapy in gastroesophageal adenocarcinoma. Patholog Res Int 2010;2011:674182.

32. Ma GF, Liu YM, Gao H, Miao Q, Luo TC, Zeng XQ, et al. HER2 mRNA status contributes to the discrepancy between gene amplification and protein overexpression in gastric cancer. Dig Dis Sci 2014;59:328-35.

33. Sartore-Bianchi A, Ardini E, Bosotti R, Amatu A, Valtorta E, Somaschini A, et al. Sensitivity to entrectinib associated with a novel LMNA-NTRK1 gene fusion in metastatic colorectal cancer. J Natl Cancer Inst 2015;108:djv306.

34. Nagasubramanian R, Wei J, Gordon P, Rastatter JC, Cox MC, Pappo A. Infantile fibrosarcoma with NTRK3-ETV6 fusion successfully treated with the tropomyosin-related kinase inhibitor LOXO-101. Pediatr Blood Cancer 2016; 63:1468-70.

35. Drilon A, Li G, Dogan S, Gounder M, Shen R, Arcila M, et al. What hides behind the MASC: clinical response and acquired resistance to entrectinib after ETV6-NTRK3 identification in a mammary analogue secretory carcinoma (MASC). Ann Oncol 2016;27:920-6.

36. Ardini E, Menichincheri M, Banfi P, Bosotti R, De Ponti C, Pulci R, et al. Entrectinib, a pan-TRK, ROS1, and ALK inhibitor with activity in multiple molecularly defined cancer indications. Mol Cancer Ther 2016;15:628-39.

37. Doebele RC, Davis LE, Vaishnavi A, Le AT, Estrada-Bernal A, Keysar S, et al. An oncogenic NTRK fusion in a patient with soft-tissue sarcoma with response to the tropomyosin-related kinase inhibitor LOXO-101. Cancer Discov 2015; 5:1049-57. 